\title{
ALCYONE - lighting the way for quadruplet therapy of NDMM
}

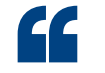

$D$-VMP is

the first

daratumumab-

based regimen

with a demon-

strated OS

benefit in

the first-line

setting

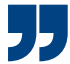

Combination therapies incorporating immunomodulatory drugs and/or proteasome inhibitors have become the standard of care for patients with newly diagnosed multiple myeloma (NDMM), including the bortezomib, melphalan and prednisone (VMP) triplet for transplant-ineligible patients. New findings support the addition of the anti-CD38 antibody daratumumab to this regimen.

In the phase III ALCYONE trial, 706 transplant-ineligible patients with NDMM were randomly assigned (1:1) to receive induction therapy with VMP and daratumumab followed by continuous maintenance treatment with daratumumab (D-VMP) or only induction VMP. In 2018, a primary analysis with a median follow-up duration of 16.5 months revealed that D-VMP improved progression-free survival (PFS), the primary end point of this study (HR 0.50, 95\% CI 0.38-0.65; $P<0.001)$. Now, updated data show that this benefit is maintained at a median follow-up duration of 40.1 months (HR $0.42,95 \%$ CI $0.34-0.51 ; P<0.0001)$; the median PFS was 36.4 months with D-VMP versus 19.3 months with VMP alone. "In addition, D-VMP substantially

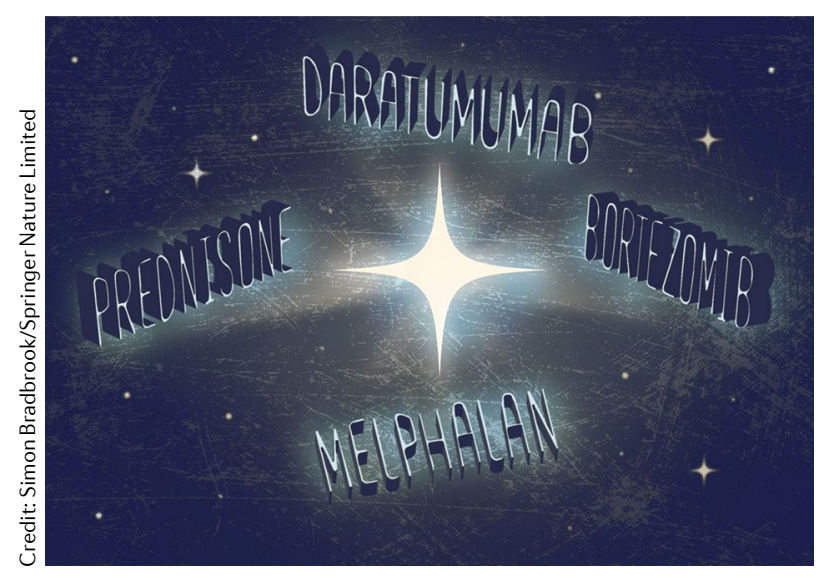

improved the complete response or better and minimal residual diseasenegative $\left(\mathrm{MRD}^{-}\right)$rates, and increased the number of patients with a $\mathrm{MRD}^{-}$ status sustained for $\geq 1$ year," explains lead author Maria-Victoria Mateos.

Importantly, these improvements translated into an overall survival (OS) benefit: 3-year OS was 78.0\% with D-VMP versus $67.9 \%$ with VMP alone (HR 0.60, 95\% CI 0.46-0.80; $P=0.0003)$. The OS hazard ratios were broadly similar across all pre-specified subgroups of patients, except those with high-risk cytogenetic features, who seemed to only modestly benefit from D-VMP (HR 0.91, 95\% CI $0.50-1.65)$; however, this subgroup was small, comprising only $14 \%$ of patients, and greater benefit might emerge with longer follow-up study "D-VMP is the first daratumumabbased regimen with a demonstrated OS benefit in the first-line setting and is a new standard of care for transplantineligible patients with NDMM," states Mateos. This treatment approach was approved by the FDA in May 2018.

Adverse event rates were similar between the treatment groups, and no new safety signals were observed with long-term daratumumab maintenance. Daratumumab was commonly associated with respiratory tract infections, in both the induction and the monotherapy periods; however, these events rarely resulted in treatment discontinuation and did not affect survival outcomes.

Patients with multiple myeloma typically require several lines of treatment. Thus, consideration of effects relating to subsequent therapies is important. In ALCYONE, PFS on the subsequent line of therapy was superior in the D-VMP group. Across most subgroups defined by type of subsequent therapy, frontline D-VMP was associated with better OS. Notably, however, only $10 \%$ of the VMP group received daratumumab in the second line, and 3-year OS was $100 \%$ in these patients, raising questions over suboptimal use of salvage therapies.

Vincent Rajkumar, who was not involved in ALCYONE, highlights other limitations: "the regimen used and the trial design make it difficult to generalize the results to clinical practice. First, only a small proportion of patients worldwide receive melphalan as initial therapy." Indeed, a triplet regimen comprising lenalidomide, bortezomib and dexamethasone (RVd), rather than VMP, is the current standard of care in the USA. "Second, daratumumab was added to induction and maintenance therapy, making it impossible to tease out where the benefit occurred." That continuous therapy improves outcomes relative to fixed-duration therapy is somewhat unsurprising.

As Rajkumar emphasizes, however, "the results of ALCYONE, in conjunction with those of the MAIA, CASSIOPEIA and GRIFFIN trials, increasingly support the use of daratumumab in the frontline setting." He adds that "the field is moving in the direction of four-drug regimens for NDMM and additional data from other ongoing trials of frontline daratumumab are eagerly awaited." These trials include a phase III study of the daratumumab plus VRd quadruplet in transplant-ineligible patients (NCT03652064).

\section{David Killock}

ORIGINAL ARTICLE Mateos, M.-V. et al. Overall survival with daratumumab, bortezomib, melphalan, and prednisone in newly diagnosed multiple myeloma (ALCYONE): a randomised, open-label, phase 3 trial. Lancet https://doi.org/10.1016/ S0140-6736(19)32956-3(2019) 